\section{A new era in Cardiogenetics}

\author{
Giuseppe Limongelli \\ Second University of Naples, \\ Monaldi Hospital, Naples, Italy
}

Unless you have insatiable curiosity about what you are studying, and the willingness to work hard to answer the questions you have posed, I think it's unlikely that you will be successful

Eugene Braunwald (Circ. Res. 2010;106;1786-1788)

Welcome to Cardiogenetics. Why Cardiogenetics? When I received the invitation to join this project my first impulse was to ask: why do we need a new journal? But the answer was there all along. Cardiogenetics is more than just the name of a new journal. Cardiogenetics is an idea.

\section{Vision}

Cardiogenetics is a new online, open access journal dedicated to general physicians, cardiologists, geneticists, and students. Cardio genetics' primary purpose is to report original findings, views, and perspectives in different basic and clinical research and translational areas, including clinical and molecular aspects of inherited and rare heart diseases in adults (curated by Prof. W.J. McKenna) and children (Prof J. Towbin), molecular genetics in cardiovascular diseases (Dr. M. Tartaglia and Dr. M. Vatta), animal models (Prof. A.J. Marian); pharmacogenetics and pharmacogenomics (Prof. M. Pirmohamed), stem cells (Prof. P. Anversa and Prof. A. Angelini), and biomarkers in cardiovascular diseases (Prof. T. Suzuki). A space will be given to negative studies in Cardiogenetics, which will be dedicated to clinical, molecular, cellular, pharmacological research studies with solid scientific background, but leading to negative results. Cardiogenetics will also welcome review articles; case reports with clinical relevance and significance, and hypotheses; methods and techniques in the field of molecular genetics, stem cells, pharmacogenomics; images in Cardiogenetics (images and/or videos dealing with unique clinical features and molecular imaging). A new series of educational articles (Education in Cardiogenetics, cured by Dr. P. Charron) will include news and views (commentary, summary, reports of outstanding articles in other journals for the general public) and interactive clinical cases. The team is completed by Raffaella D'Alessandro and Valeria Maddaloni (Local Board), Paola Granata (Managing Editor) and Cristiana Poggi (Layout Editor). They represent the soul of this new journal, and they work side by side with the Editor-in-Chief to put each single piece of the puzzle in the right place.

\section{Is Cardiogenetics timely?}

The birth of cardiovascular genetics

In 1989, the Seidman Lab reported the first association between an inherited gene defect and a primary cardiomyopathy. ${ }^{1}$ In a subsequent multicentre study involving European and US scientists, they reported the first $\beta$ myosin missense mutation in a FrenchCanadian family with hypertrophic cardiomyopathy (HCM), leading to the equation one gene $=$ one disease. That seminal observation represented a dividing line between the previous (old) and the new (current) cardiology, and the beginning of cascade of discoveries.

From One Gene-One Disease to One DiseaseMany Genes. .

Since then, substantial progress has been made in further elucidating the genetic background of cardiomyopathies. More than 450 mutations affecting genes coding for sarcomeric proteins (thick and thin filaments) have been identified in patients with HCM, establishing HCM as a disease of the sarcomere. At the same time, dilated cardiomyopathy (DCM) was indicated as a disease of the cytoskeleton and extracellular matrix, while arrhythmogenic cardiomyopathy (ARVC) as a disease of the desmosomes, following the final common pathway hypothesis delineated by Towbin and colleagues in the late $1990 \mathrm{~s}^{2}$

\section{To One Disease-Many Different Genes...}

However, this view based on functional modules was challenged by further genetic discoveries showing that those definitions failed to account for disease in many patients. It is now recognized that mutations in sarcomeric genes account for approximately $50-60 \%$ of all cases of HCM, and that rare causes of HCM have been associated with mutations in sarcomere-related protein genes (i.e., myosin light chain kinase, muscle LIM protein, telethonin, titin, etc.), or genes coding for proteins with different role in intracellular signaling (phospholamban, RAF1).

\section{... To One Gene-Many Diseases}

Evidence supporting the view that a family of functionally related genes (i.e., sarcomeric protein genes) may be responsible for a spectrum of different phenotypes (HCM, DCM, the restrictive and the left ventricular noncompaction phenotype), represents a leap in our understanding of cardiomyopathies. However, whether this means that these are different diseases or rather different manifestations
Correspondence: Giuseppe Limongelli,

Second University of Naples, Monaldi Hospital, Naples, Italy.

E-mail: giuseppe.limongelli@cardiogenetics.it

Key words: cardiogenetics, next generation sequencing, OMICS.

Received for publication: 21 June 2011. Accepted for publication: 30 June 2011.

This work is licensed under a Creative Commons Attribution NonCommercial 3.0 License (CC BYNC 3.0).

(C) Copyright G. Limongelli, 2011

Licensee PAGEPress, Italy

Cardiogenetics 2011; 1:e1

doi:10.4081/cardiogenetics.2011.e1

(phenotypes) of the same pathological mechanism is still not clear. An emblematic case is SCN5A (sodium channel) gene mutations. It may cause phenotypes that combine features of Long QT Syndrome 3 (LQT3), Brugada syndrome, conduction disease and dilated cardiomyopathy (one gene-different diseases), and represent the link between the world of cardiomyopathies (heart muscle diseases) and that of ion channel diseases (heart conduction disease and arrhythmias) as predicted in the 2006 AHA consensus regarding contemporary definitions and classification of cardiomyopathies. $^{3}$

Does this knowledge have any practical impact? Genomic medicine has entered clinical practice, and the recognition of the diagnostic utility of genetic testing for cardiomyopathies is growing. With expanding knowledge of the genetic background of these diseases, primary cardiomyopathies have recently been subclassified into genetic, mixed, and acquired disease (AHA 2006) or familial and non familial diseases (ESC 2007), shifting the general view of cardiomyopathies toward a new model including both phenotype and genotype, phenocopies and genocopies, but with the phenotype as a starting point of the diagnostic approach., ${ }^{3,4}$

\section{What does the future hold?}

Over the last decades, a growing body of knowledge acquired on all the aspects of human and molecular genetics has changed our general feeling for cardiovascular diseases. The Human Genome Project has been the largest and most important discovery in this field. Indeed, millions of sequence changes have already been discovered across the human genome, so far. Such variation includes germline and acquired lesions that differ widely by type and size (from singlebase mutations to complex chromosome 
rearrangements and variation in copy number (CNVs). Nevertheless, the search of the molecular mechanisms underlying cardiovascular disease is in its early stage. The paradigm one gene=one disease is severely challenged. A complex interplay of genetic and environmental factors have been advocated in different monogenic disorders, such as familial pulmonary hypertension. A multi-hit hypothesis, including the effects of different susceptibility genes involved in the process of cardiogenesis associated to environmental factors, have been proposed to explain the enormous variability observed in familial cluster of non-syndromic congenital heart diseases. Moreover, discovery of genomic regions and genetic polymorphisms that influence susceptibility to common complex diseases, as coronary artery disease, is also accelerating. Several challenges still exist in this field, including phenotype and genotype heterogeneity, gene-gene and gene-environment interactions, and the fact that the etiologic spectrum ranges from common genetic variants with small effects to rare genetic variants with large effects. The identification of causal genes for these complex diseases requires a robust multidisciplinary approach that includes physicians, scientists, epidemiologists and statisticians with expertise in genetics.
The development of new high throughput DNA sequencing technology (next generation sequencing, NGS) and newer phenotyping and analytical methods show promise for accelerating progress in this field. ${ }^{5}$ NGS represents today the technology of choice in many areas of biomedical research, particularly for complex genetic analyses that were previously either technically infeasible or cost prohibitive. Conversely, the dissemination of NGS in clinical diagnostics is in its infancy.

\section{GROW}

On this ground, we strongly believe that the development of a new journal dedicated to cardiovascular genetics is timely. One acronym sometime used for problem solving is GROW. Goals, Reality, Options, and Will. Our principal Goals for Cardiogenetics are: i) to connect the scientific (bench) to the clinical (bedside) world; ii) to become an essential resource and Reality to students, general practitioners, cardiology fellows, clinical cardiologists, clinical and molecular geneticists. Indeed, the education of geneticists in cardiology and, conversely, the education of physicians and cardiologists in system biology (the so called OMICS) is erroneously considered just an option. Cardiogenetics has the Will to change this paradigma.

Welcome to Cardiogenetics.

\section{References}

1. Seidman CE, Seidman JG. Identifying sarcomere gene mutations in hypertrophic cardiomyopathy: a personal history. Circ Res 2011;108:743-50.

2. Bowles NE, Bowles KR, Towbin, JA. The final common pathwaym hypothesis and inherited cardiovascular disease. The role of cytoskeletal proteins in dilated cardiomyopathy. Herz 2000;25:168-75.

3. Maron BJ, Towbin JA, Thiene G, et al. Contemporary definitions and classification of the cardiomyopathies: an American Heart Association Scientific Statement from the Council on Clinical Cardiology, Heart Failure and Transplantation Committee; Quality of Care and Outcomes Research and Functional Genomics and Translational Biology Interdisciplinary Working Groups; and Council on Epidemiology and Prevention. Circulation 2006;113:1807-16.

4. Elliott P, Andersson B, Arbustini E, et al. Classification of the cardiomyopathies: a position statement from the European Society Of Cardiology Working Group on Myocardial and Pericardial Diseases. Eur Heart J 2008;29:270-6.

5. Schuster SC. Next-generation sequencing transforms today's biology. Nature Methods 2008;5:16-8. 